\title{
Dermatitis Neglecta: An oft Missed Diagnosis
}

\author{
Sonia Agrawal ${ }^{1, *}$, and Vijay Gandhi' \\ ${ }^{1}$ Post Graduate Resident, Department of Dermatology \& STD, UCMS \& GTB hospital, Delhi, India \\ ${ }^{2}$ Professor, Department of Dermatology \& STD, UCMS \& GTB hospital, Delhi, India
}

*Corresponding author: Agrawal S, Post Graduate Resident, Department of Dermatology \& STD, UCMS \& GTB hospital, Delhi, India, Tel: 09489429039 ; E-mail: sonia.03agrawal@gmail.com

Received: 24 Feb, 2020 | Accepted: 24 Mar, 2020 | Published: 31 Mar, 2020

Citation: Agrawal S, Gandhi V (2020) Dermatitis Neglecta: An oft Missed Diagnosis. J Clin Cosmet Dermatol 4(2): dx.doi.org/10.16966/25762826.149

Copyright: @ 2020 Agrawal S, et al. This is an open-access article distributed under the terms of the Creative Commons Attribution License, which permits unrestricted use, distribution, and reproduction in any medium, provided the original author and source are credited.

\begin{abstract}
Dermatitis neglecta, also known as 'dirty dermatosis' or 'unwashed dermatosis' is an uncommon yet noteworthy entity as it can be easily misdiagnosed for many other dermatoses [1]. This entity was first reported by Poskitt L, et al. [2]. The probable cause is lack of proper cleansing of skin leading to accumulation of sweat, sebum, and exfoliated corneocytes with growth of micro-organisms. This results in formation of a layer of adherent scale crust over the affected part [3]. In some patients underlying disorders like psychiatric illness [4] or neurological diseases [5] have been described. Clinical presentation is in the form of hyperkeratotic verrucous plaques mimicking a variety of specific dermatoses like seborrheic dermatitis, pemphigus foliaceous, verrucous epidermal naevus, darier's disease and terra firma forme dermatoses [4].
\end{abstract}

We report two cases presenting to the outpatients department with classical presentation.

\section{Case I}

A young girl presented with hyperkeratotic, yellow to brown crusted plaques overmalar area of face for 1 month. The lesions were asymptomatic but caused significant social, cosmetic and psychological problems. Cutaneous examination revealed multiple, yellowish brown, crusted plaques predominantly over the forehead, malar and perioral areas with sparing of eyelids and peri-orbital areas (Figure 1). The scales appeared characteristically like corn flakes. Further, on rubbing the plaques gently with moist cotton, the crusts were easily removable with underlying skin showing normal morphology. A detailed history was taken with special stress on measures adopted to clean the face. It was revealed that she had not been using any soap or exfoliating agent to clean her face due to apprehension that these agents cause roughening of the face. A clinical diagnosis of dermatitis neglecta was made and the patient was counselled to wash her face regularly with soap and water .After 1 week, there was a dramatic response with almost complete clearance of lesions (Figure 2).

\section{Case II}

A 42 year old male, who was a known case of paranoid schizophrenia was referred from a nearby hospital with a provisional diagnosis of psoriasis vulgaris. On examination, dirty yellow crusted plaques were seen over the bilateral malar areas involving the dorsum of nose and forehead (Figure 3). There were no lesions elsewhere in the body. A provisional diagnosis of dermatitis neglecta was made. On interrogation, he admitted that there was self-neglect in cleaning the face due to mood disturbances. He was not using any soap or face wash and occasionally cleaned with water only. On gently scrubbing the lesions over forehead with moist gauze, the crusts came off easily leaving behind normal skin (Figure 4). Patient was counselled regarding facial cleansing hygiene and prescribed face cleanser.

Risk factors for dermatitis neglecta include, chronic disability, old age, sensitive skin, trauma and mental health conditions. In all these conditions, either there is loss of self-care or the disease per say prevents the patients from cleaning the affected areas. The closest differential diagnosis of dermatitis neglecta includes terra firma forme dermatoses or 'Duncan's dirty dermatoses' [6]. A considerable overlap exists between these two dermatoses. There is presence of asymptomatic dirty brown plaques despite satisfactory hygiene $[7,8]$. Also the patients lack the characteristic corn-flake like scales seen in dermatitis neglecta. Other hyperpigmented conditions like the dirty neck appearance of atopic dermatitis or confluent and reticulate papillomatosis are resistant to scrubbing with moist gauze. Dermatitis artefacta is a close mimicker seen in patients with perosnality disorders, wherein there is an act of commission rather than omission [9]. Patients have the tendency of self-infliction of lesions which appear dirty and hyperpigmented. However, there is absence of the characteristic features of dermatitis neglecta. Other common differential diagnoses include pityriasis versicolor, ichthyosis, verrucous naevi, pemphigus foliaceous and darier's disease [8].

\section{Conclusion}

Dermatitis neglecta is predominantly a clinical diagnosis with 


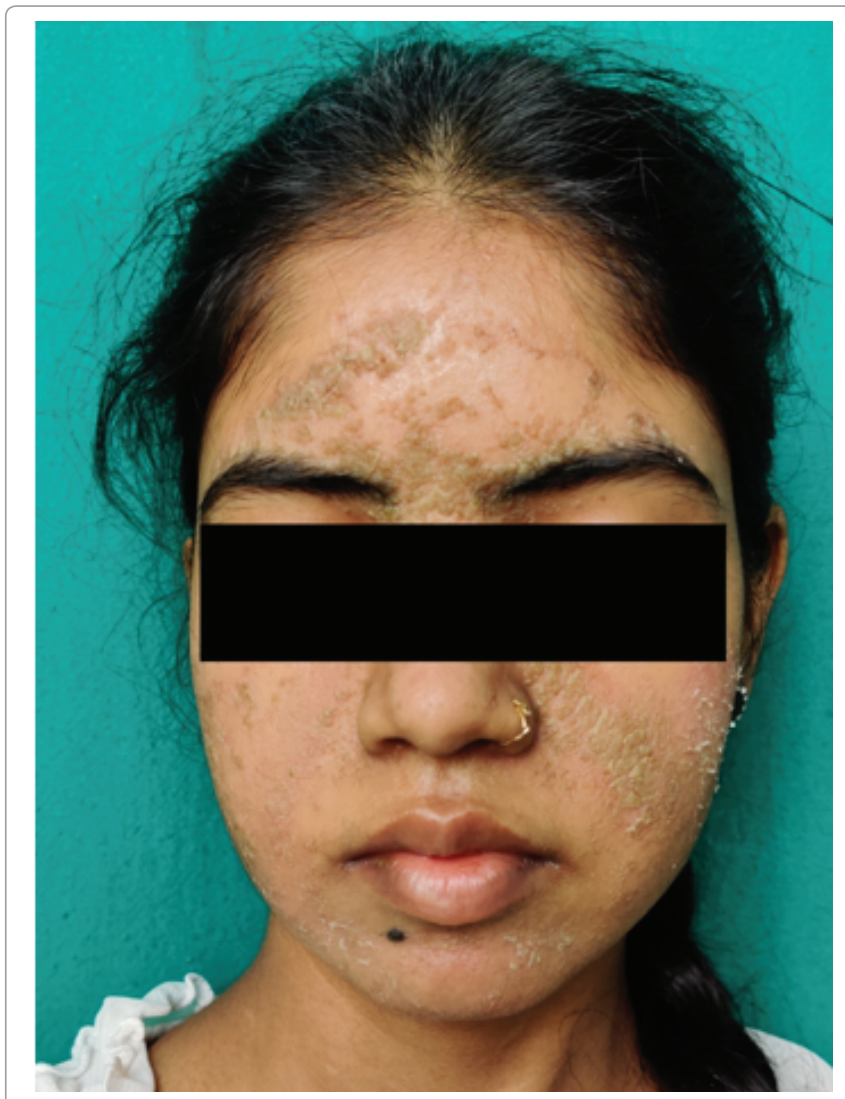

Figure 1: Presence of multiple, yellow brown crusted plaques appearing like corn flakes.

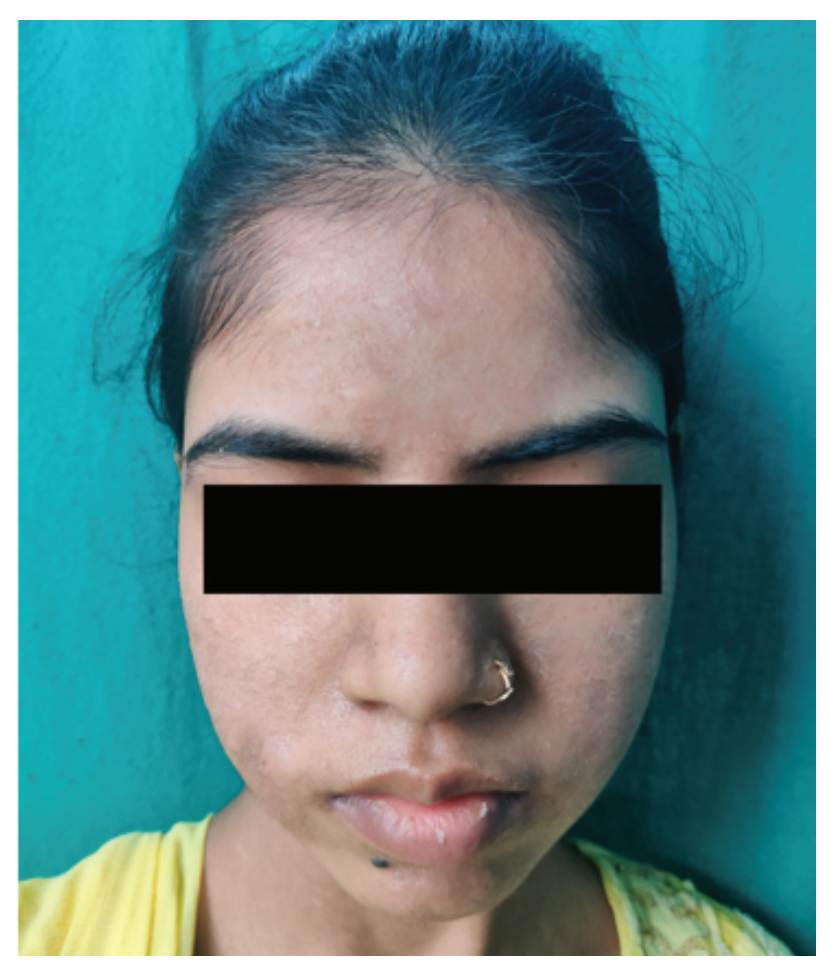

Figure 2: Clearance of lesions after one week of regular face wash with soap and water.

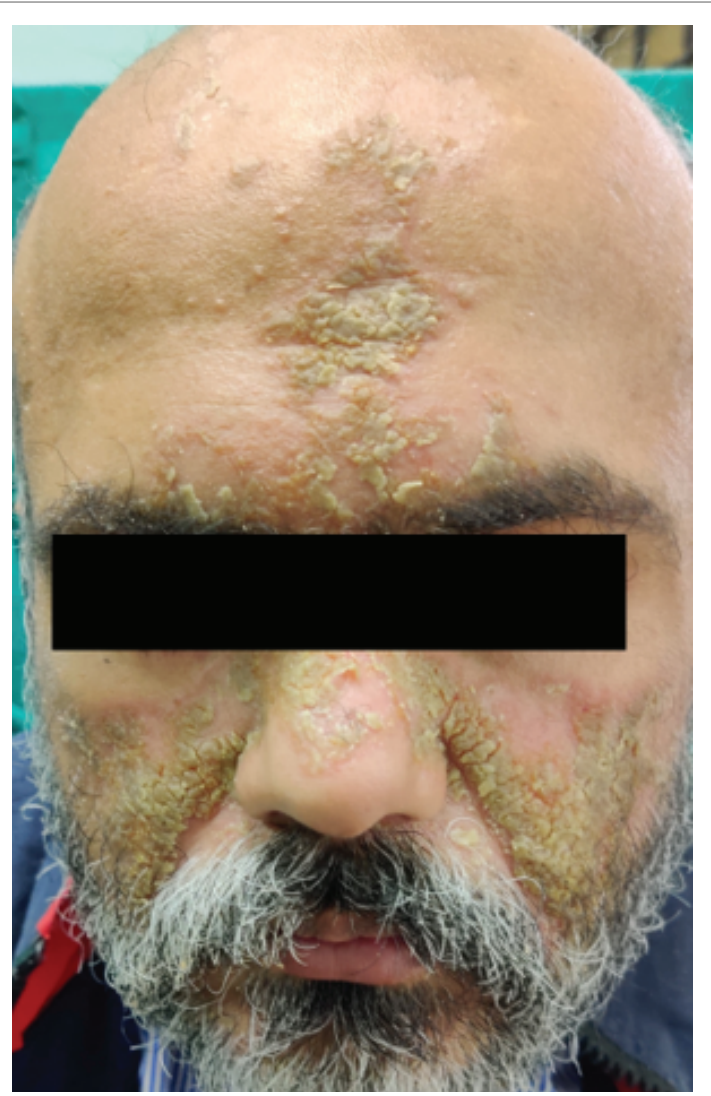

Figure 3: Presence of multiple, yellow brown crusted plaques appearing like corn flakes.

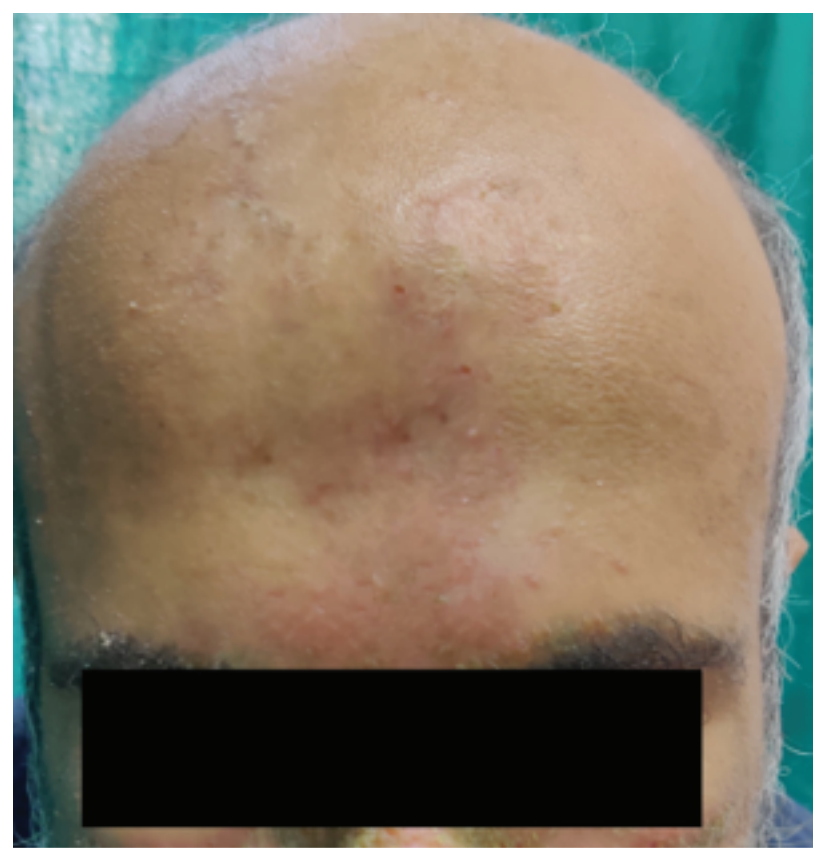

Figure 4: Clearance of lesions over forehead on wiping with alcohol soaked cotton gauze. 
high index of suspicion and requires no specific diagnostic test or histopathology. Diagnosis can be easily established by pertinent history taking, presence of characteristic corn-flake like scales, and easy removal of the scales using alcohol soaked gauze. Thus, the moist gauze acts both as a diagnostic as well as therapeutic modality. Treatment includes proper counselling and maintenance of hygiene. In resistant cases, emollients or keratolyic agents like 6\% salicylic acid can be advocated. Response to treatment is good with a satisfactory aesthetic appearance.

Thus, dermatitis neglecta should be considered in differential diagnosis of patients presenting with crusted and hyperkeratotic lesions on face especially in absence of involvement of other sites .Clinical suspicion can be easily confirmed by cleansing the skin with moist swab. This may avoid unwarranted investigations including skin biopsy. Treatment includes proper counselling and cleaning the affected area with simple measures like alcohol-soaked gauze, soap and water. This is especially important for young dermatologists who can easily overlook an easily treatable dermatosis.

\section{Conflict of Interest}

None

\section{Sources of Support}

None

\section{Authors' Contribution}

Dr. Sonia Agrawal first saw the case, took clinical images and counselled and treated the patient under supervision of Dr Vijay
Gandhi. She also drafted the article. Dr. Vijay Gandhi, made the diagnosis, supervised management and gave substantial inputs on the drafting and revision of the file.

\section{References}

1. Saha A, Seth J, Sharma A, Biswas D (2015) Dermatitis Neglecta-A dirty dermatosis: Report of Three Cases. Indian J Dermatol 60: 185187.

2. Poskitt L, Wayte J, Wojnarowska F, Wilkinson JD (1995) "Dermatitis Neglecta": Unwashed Dermatosis. Br J Dermatol 132: 827-829.

3. Saritha M, Karthikeyan K (2015) Dermatitis neglecta-to be remembered, not neglected! Indian Dermatol Online J 6: 138-139.

4. Pérez-Rodríguez IM, Muñoz-Garza FZ, Ocampo-Candiani J (2014) An Unusually Severe Case of Dermatosis Neglecta: A Diagnostic Challenge. Case Rep Dermatol 6: 194-199.

5. Choudhary S, Bisati S, Koley S (2011) Dermatitis Neglecta. Indian J Dermatol Venereol Leprol 77: 62-63.

6. Aslan NÇ, Güler Ş, Demirci K, Isiyel E (2018) Features of Terra FirmaForme Dermatosis. Ann Fam Med 16: 52-54.

7. Langar S, Sonthalia S (2018) Dermatitis Neglecta. Indian J Med Res 147: 322.

8. Erkek E, Sahin S, Çetin ED, Sezer E (2012) Terra Firma-Forme Dermatosis. Indian J Dermatol Venereol Leprol 78: 358-360.

9. Saha A, Seth J, Gorai S, Bindal A (2015) Dermatitis Artefacta: A Review of Five Cases: A Diagnostic and Therapeutic Challenge. Indian J Dermatol 60: 613-615. 